\title{
PRL-3 promotes the proliferation of LoVo cells via the upregulation of KCNN4 channels
}

\author{
WEI LAI ${ }^{*}$, SHUANG CHEN ${ }^{1 *}$, HENG WU $^{1}$, YUFENG GUAN ${ }^{1}$, LU LIU $^{1}$, YUJIE ZENG ${ }^{1}$, \\ HAIYAN ZHAO ${ }^{2}$, JIANMIN JIANG ${ }^{3}$ and ZHONGHUA CHU ${ }^{1}$ \\ Departments of ${ }^{1}$ Gastroenteropancreatic Surgery and ${ }^{2}$ Medical Research Center, Sun Yat-sen Memorial \\ Hospital, Sun Yat-sen University, Guangzhou 510120; ${ }^{3}$ Department of Pharmacology and Toxicology, \\ School of Pharmaceutical Sciences, Sun Yat-sen University, Guangzhou 510006, P.R. China
}

Received May 5, 2011; Accepted May 31, 2011

DOI: $10.3892 /$ or.2011.1366

\begin{abstract}
Previous studies have shown that phosphatase of regenerating liver-3 (PRL-3) plays an important role in the metastasis and proliferation of tumor cells. However, the mechanism by which PRL-3 controls the cell cycle of tumor cells remains unknown. In the present study, considering that the $\mathrm{K}^{+}$channels strictly control cell proliferation, we examined whether $\mathrm{K}^{+}$ channels participate in the proliferation of tumor cells induced by PRL-3. Interestingly, the expression of intermediate-conductance $\mathrm{Ca}^{2+}$-activated $\mathrm{K}^{+}$channels $(\mathrm{KCNN} 4)$ was upregulated in an NF- $\kappa \mathrm{B}$-dependent manner when PRL-3 was transfected into LoVo cells. Also, we identified two NF- $\kappa \mathrm{B}$ binding sites in the promoter region of KCNN4. Use of the specific inhibitor 1-[(2-chlorophenyl) diphenylmethyl]-1H-pyrazole (TRAM-34) significantly inhibited the proliferation induced by PRL-3 and blocked the cell cycle at the G2/M phase. Meanwhile, the level of phosphorylation of $\mathrm{Cdc} 2$ was increased in a dose-dependent manner. Furthermore, TRAM-34 also inhibited tumor formation of PRL-3 cell xenografts implanted by injection in nude mice. In conclusion, PRL-3 promoted the proliferation of LoVo cells through upregulation of KCNN4 channels which facilitated the $\mathrm{G} 2 / \mathrm{M}$ transition.
\end{abstract}

\section{Introduction}

Currently, colorectal cancer is one of most common malignancies worldwide. In terms of its incidence rate, it has ranked third among the most common cancers. In 2009, there were 146,970 new cases and 49,920 deaths due to the disease in the United States (1). The survival rate for patients with liver metastasis is

Correspondence to: Professor Zhonghua Chu, Department of Gastroenteropancreatic Surgery of the Sun Yat-sen Memorial Hospital, Sun Yat-sen University, Guangzhou 510120, P.R. China E-mail: chuzhonghua2010@yahoo.cn

\section{${ }^{*}$ Contributed equally}

Key words: phosphatase of regenerating liver-3, KCNN4, Cdc2, $\mathrm{NF}-\kappa \mathrm{B}$
$37 \%$ (2). Among the molecular mechanisms leading to colorectal metastasis, PRL-3 is of interest as it has been shown to be overexpressed in liver metastases of colorectal cancer, but seldom expressed in primary tumors and normal colorectal epithelium (3). PRL-3 belongs to the protein-tyrosine phosphatases (PTPs) family which plays an important role in signal transduction (4). Researchers have pointed out that PRL-3 may have a role in the metastases of cancer other than colorectal cancer. For example, overexpression of PRL-3 has been associated with metastasis of gastric cancer and human myeloma cells $(5,6)$. In addition, small interfering RNA significantly inhibited the metastasis of B16-BL6 mouse melanoma cells and gastric cancer cells $(7,8)$. Apart from that, PRL-3 has been proven to promote the growth of ovarian cancer (9). Previous research has revealed that the proliferation of pancreatic ductal epithelial cells was induced by PRL-1 and PRL-2 through downregulation of cyclin-dependent kinase inhibitor $\mathrm{p} 21^{\mathrm{Cip} 1 / \mathrm{Waf} 1}(10)$. However, the mechanism by which PRL-3 promotes the proliferation of cancer cells remains unknown. In summary, the above mentioned studies suggest that PRL-3 may regulate a pathway critical for tumorigenesis and metastasis and thus support that the underlying mechanism of the PRL-3-mediated proliferation of tumor cells should be further explored.

Previous studies have also revealed that $\mathrm{K}^{+}$channels regulate the cell cycle progression and are involved in cell growth which indicates that they may be a new strategy for cancer therapy (11). Additional research has indicated that $\mathrm{K}^{+}$channels control intracellular $\mathrm{pH}$ and $\mathrm{Ca}^{2+}$ signaling to influence proliferation (12). KCNN4 channels are prominently expressed in cells of the hematopoietic system. KCNN4 belongs to the $\mathrm{Ca}^{2+}$-activated $\mathrm{K}^{+}$channel superfamily whose activation is $\mathrm{Ca}^{2+}$-dependent, but not time or voltage dependent (13). Previous researchers have demonstrated that KCNN4 channels play an important role in the immune system (14). Up until now, KCNN4 channels have also been found to support the proliferation of tumor cells including prostate cancer cells and a specific inhibitor has been shown to prevent the growth induced by upregulation of KCNN4 channels (15).

In the present study, the effect of KCNN4 channels on the PRL-3-induced proliferation was examined in LoVo cells. Once LoVo cells were transfected with PRL-3, the expression of KCNN4 channels was upregulated in a NF- $\mathrm{KB}$-dependent 
manner. The specific inhibitor TRAM-34 indicated that the PRL-3-induced growth of LoVo cells could be suppressed not only in vivo but also in vitro.

\section{Materials and methods}

Materials. Rabbit polyclonal antibody specific for PRL-3 and TNF- $\alpha$ were purchased from Abcam, polyclonal anti-KCNN4 antibody, polyclonal anti-p-Cdcp34(Thr14/Tyr15) antibody, monoclonal anti-Cdc2 p34 antibody and anti-rabbit/mouse IgG-conjugated horseradish peroxidase (HRP) were purchased from Santa Cruz Biotechnology. TRAM-34, DMSO and thymidine were purchased from Sigma-Aldrich and 1-ethyl2-benzimidazolinone (1-EBIO) was purchased from Tocris.

Cell culture and synchronization. The human colon cancer cell line LoVo was purchased from the Shanghai Cell Bank of the Chinese Academy of Sciences and maintained in RPMI-1640 supplemented with 10\% fetal bovine serum (Gibco), $100 \mathrm{IU} / \mathrm{ml}$ penicillin, and $100 \mu \mathrm{g} / \mathrm{ml}$ streptomycin. The culture flasks were incubated at $37^{\circ} \mathrm{C}$ at a $5 \% \mathrm{CO}_{2}$ humidified atmosphere. The media were changed every second or third day. Cells were synchronized at the G1/S phase boundary using the double thymidine blockade method (16). Briefly, to synchronize cells at the G1/S boundary, cells were incubated in medium containing $2 \mathrm{mM}$ thymidine for $16 \mathrm{~h}$ followed by an 8 -h recovery and then the cells were synchronized at the G1/S boundary with $2 \mathrm{mM}$ thymidine for an additional $16 \mathrm{~h}$.

Construction of PAcGFP-PRL-3 plasmid and stable clone selection. Specific primers for PRL-3 (GenBank accession no. NM032611) were synthesized as follows: PRL-3/forward, 5'-CCGCTCGAGATGGCTCGGATGAACC-3'; PRL-3/ reverse, 5'-CGGAATTCCTACATAACGCAGCACCG-3'. To obtain the coding region of PRL-3, RT-PCR was performed using the PrimeScript RT-PCR kit and the product were retrieved by the DNA fragment purification kit (Takara) followed by XhoI and EcoRI digestion. Finally, human PRL-3 was inserted in the corresponding sites of PAcGFP-C3 (Clontech). Transfection was performed using Lipofectamine 2000 (Invitrogen) according to the manufacturer. After transfection, selective media were used containing $600 \mu \mathrm{g} / \mathrm{ml} \mathrm{G} 418$ (Sigma-Aldrich) until the stable clone was picked up after 3 weeks.

Reverse transcription and real-time polymerase chain reaction. Total RNA was extracted using the TRIzol reagent (Invitrogen) according to the manufacturer's protocol. The first-strand cDNA was synthesized from 500 ng RNA using the PrimeScript RT reagent kit. Then, quantitative real-time RT-PCR was performed using the LightCycler 480 (Roche Diagnostics) and the SYBR assays (Takara). The primers designed to detect PRL-3 (GenBank accession no. NM032611), TNF- $\alpha$ (GenBank accession no. NM000594) and KCNN4 (GenBank accession no. NM002250) were as follows: PRL-3/ forward, CGCTTATTGAGAGCGGGATGA; PRL-3/reverse, TGAACCGCAGCCTCTGTTTG; TNF- $\alpha /$ forward, GACA AGCCTGTAGCCCATGTTGTA; TNF- $\alpha /$ reverse, CAGCCT TGGCCCTTGAAGA; KCNN4/forward, GAAGCTCCGG GAACAAGTGAAC; KCNN4/reverse, CTCAGATTCTGC TGCAGGTCATACA. As an internal control, the expression of the GAPDH gene (GenBank accession no. NM002046) was detected using the following primers: GAPDH/forward, GCA CCGTCAAGGCTGAGAAC; GAPDH/reverse, TGGTGAAG ACGCCAGTGGA. According to the manufacturer's instructions, each sample contained the following components at the indicated concentration in a final volume of 20:10 $\mu \mathrm{l}$ of $2 \mathrm{X}$ SYBR Premix Ex Taq ${ }^{\mathrm{TM}}, 0.2 \mu \mathrm{M}$ of each forward and reverse primers and $500 \mathrm{ng}$ template cDNA. Subsequently, for each reaction the parameters were set as follows: the mixture were initially denatured at $95^{\circ} \mathrm{C}$ for $5 \mathrm{sec}$, followed by 40 cycles of amplification $\left(95^{\circ} \mathrm{C}\right.$ for $5 \mathrm{sec}$ and $60^{\circ} \mathrm{C}$ for $\left.20 \mathrm{sec}\right)$. For the relative quantification, $2^{-\Delta \Delta \mathrm{Ct}}$ was used to calculate the fold-change in gene expression. All of the experiments were performed in triplicate.

Protein extraction and Western blotting. LoVo cell cultures were washed with PBS and lysed with RIPA containing (mM) 50 Tris- $\mathrm{HCl}$ (pH 7.4), $150 \mathrm{NaCl}, 0.5$ PMSF, $1 \%$ Triton X-100, $1 \%$ sodium deoxycholate and $0.1 \%$ SDS. Protein concentration was determined by Bradford assay, and then equal amounts of protein $\left(30 \mu \mathrm{g} /\right.$ lane) were boiled at $100^{\circ} \mathrm{C}$ with loading buffer for 5 min and separated by electrophoresis on $12 \%$ SDS-PAGE gels. The proteins were transferred to nitrocellulose polyvinylidene difluoride (PVDF) membranes (Bio-Rad) and then blocked in TBS with 5\% non-fat milk. The blots were further incubated with the following primary antibodies overnight at $4^{\circ} \mathrm{C}$ : polyclonal anti-PRL-3 antibody, polyclonal anti-KCNN4 antibody, polyclonal anti-TNF- $\alpha$ antibody, polyclonal anti-p-Cdc2 p34(Thr14/ Tyr15) antibody and monoclonal anti-Cdc2 p34 antibody. After washing, the membranes were incubated with HRP-conjugated secondary antibody (1:5000) for $1 \mathrm{~h}$ at room temperature. Labelled proteins were visualized by enhanced chemiluminescence reagents (Amersham Biosciences) and developed on X-ray film. The expression of GAPDH was used as an internal control to confirm the equivalence of the protein concentration in each sample with anti-GAPDH antibody. The relative band intensities of blots were measured by the Quantity One software (Bio-Rad).

Electrophoretic mobility shift assay. The nuclear proteins of the control vector cells and PRL-3 cells were extracted using a Nuclear Extraction kit (Promega) according to the manufacturer's instructions. A total of $5 \mu \mathrm{g}$ of nuclear protein were incubated with biotin-labeled DNA probes $(200 \mathrm{nM})$ for $10 \mathrm{~min}$ at room temperature in a final volume of $20 \mu \mathrm{l}: 50 \%$ glycerol, $10 \mathrm{X}$ binding buffer, $1 \mathrm{mM} \mathrm{MgCl}_{2}, 0.1 \%$ Nonidet P-40 and $50 \mu \mathrm{g} / \mathrm{ml}$ of poly (dI-dC). Protein-DNA complexes were separated from the free DNA probe through 5\% non-denaturing polyacrylamide gels in $0.5 \mathrm{X}$ TBE buffer and gels were dried and analyzed by the Streptavidin-HRP detection kit (BioLab). According to the two putative NF- $\mathrm{KB}$ binding sites located at the promoter region of KCNN4 (indicated by underlined primers), two putative $\mathrm{NF}-\kappa \mathrm{B}$ binding probes were synthesized as follows: 1F: 5'-CCCCCTACCCTGGGGCC TCCCCTGCCCCTCCCC-3'; 1R: GGGGAGGGGCAGGG GAGGCCCCAGGGTAGGGGG-3'; 2F: 5'-GGCTACTATA GGTATGTCCCTAAATGTTGT-3'; 2R: 5'-ACAACATTTA GGGACATACCTATAGTAGCC-3'.

Cell proliferation assay. Considering the disadvantages of MTT compared with WST-8, which can be reduced to soluble 


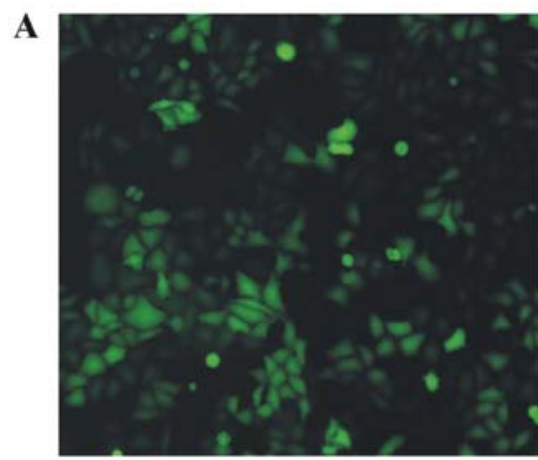

PRL-3

B

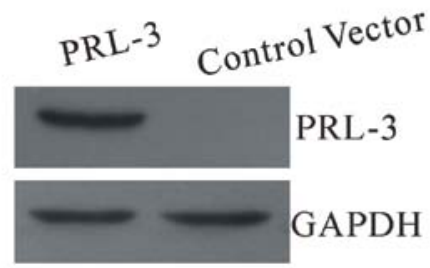

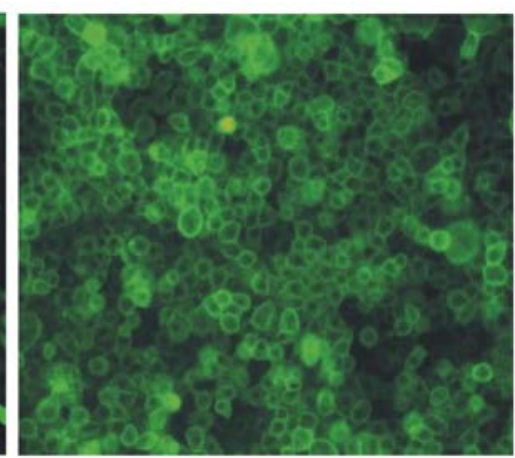

Control Vector

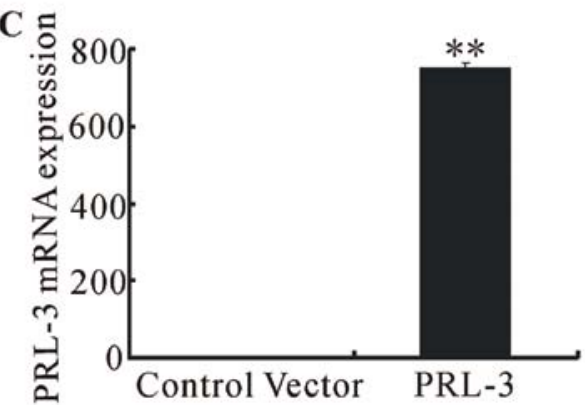

Figure 1. The expression of PRL-3 in control vector cells and PRL-3 cells. (A) LoVo cells stably transfected with PAcGFP-C3 (left) and PAcGFP-C3-PRL3 (right) were established. (B) Cell lysates $(30 \mu \mathrm{g})$ from stable cell lines were used to confirm PRL-3 expression by immunoblotting with anti-PRL-3 antibody. The antibody to the GAPDH was also used to probe for GAPDH as a loading control. The expression of ectopic PRL-3 can only be detected in PRL-3 cells (left). (C) PRL-3 mRNA was determined by real-time quantitative RT-PCR. GAPDH mRNA was used as an internal control. The means and SD are representative of three independent experiments. ${ }^{* *} \mathrm{p}<0.01$, compared with control.

formazan by yellow-colored dehydrogenases in cells and has little toxicity to cells, cell proliferation was measured using the Cell Counting kit-8 (CCK-8) assay according to manufacturer's instructions. Cells were suspended at a final concentration of $5 \times 10^{5}$ cells/well and seeded in 96-well flat-bottomed microplates. Subsequently, the cells were cultured at $37^{\circ} \mathrm{C}$ in a $5 \% \mathrm{CO}_{2}$ humidified atmosphere for $24 \mathrm{~h}$. After that, media containing TRAM-34 and DMSO at increasing concentrations were used for $24 \mathrm{~h}$ and $10 \mu \mathrm{l}$ CCK- 8 was added into each well. The absorbance was determined at a $450 \mathrm{~nm}$ wavelength with a reference wavelength of $630 \mathrm{~nm}$ after incubation of the cells for $1 \mathrm{~h}$ at $37^{\circ} \mathrm{C}$.

Flow cytometry analysis. Cells exposed to TRAM-34 and DMSO at different concentrations for $24 \mathrm{~h}$ were fixed with $2 \mathrm{ml}$ ice-cold $70 \%$ ethanol overnight at $4^{\circ} \mathrm{C}$ followed by three washes with PBS and propidium iodide staining (Sigma) at the final concentration of $0.05 \mathrm{mg} / \mathrm{ml}$. The cell cycle status was measured by flow cytometry (Becton-Dickson) through 10,000 events and the ModFit LT 2.0 ${ }^{\mathrm{TM}}$ software (Verity Software House Inc., Topsham, ME) was used to assess the cell cycle distribution patterns (G0/G1, S and G2/M phases).

Patch-clamp experiments. The patch-clamp technique was performed in the whole-cell configuration using an Axoclamp 200B amplifier (Axon Instruments). The internal pipette solution for whole cell current recordings in LoVo cells contained (in $\mathrm{mM}$ ) $140 \mathrm{KCl}, 2 \mathrm{MgCl}_{2}, 10 \mathrm{HEPES}, 2 \mathrm{Na}^{+} \mathrm{ATP}, 0.1 \mathrm{GTP}$, (pH 7.3). The standard extracellular solution contained (in $\mathrm{mM}$ ) $140 \mathrm{NaCl}, 5 \mathrm{KCl}, 2 \mathrm{CaCl}_{2}, 20$ HEPES and 10 glucose, $\mathrm{pH}$ adjusted to $7.4(\mathrm{NaOH})$. For whole cell currents recording, voltage stimuli lasting $250 \mathrm{msec}$ were delivered at $1 \mathrm{sec}$ intervals, with voltage ramps ranging from -150 to $+150 \mathrm{mV}$. The currents were digitized (sampled at a frequency of $10 \mathrm{kHz}$ ) and stored on computer, and then subsequently analyzed using the pClamp 10.0 software (Axon Instruments). Capacitance transients were minimized using the capacitance neutralization circuits on the amplifier. Correction for series resistance was not routinely applied. According to the indicated concentrations, corresponding drugs were added directly to the recording chamber. Experiments were performed at room temperature of $27^{\circ} \mathrm{C}$.

Mouse tumor model. Athymic nude mice (BALB/c nu/nu, 3-week old females) were purchase from the Vital River Laboratories (China). A total of 49 mice were randomly divided into seven groups (7 mice/group). Mice in the treatment groups were injected with LoVo cancer cells trasfected with the PAcGFP-PRL-3 vector $\left(5 \times 10^{6}\right.$ in $\left.100 \mu \mathrm{l}\right)$ and pretreated with TRAM-34 at different concentrations at the right flank. For mice in the control groups, the same number of cells treated with corresponding concentration of DMSO were injected at the same place. Tumor weight and volume were recorded every third day. Tumor volume was calculated according to the following equation: $V=a \times b^{2} / 2$, where $a$ is the length and $b$ is the width of a tumor measured by caliper. On Day 21, all animals were sacrificed. Tumors were taken out and weights were measured with a scale.

Statistical analysis. Statistical analyses were performed using SPSS 13.0 (SPSS Inc., USA). All data are presented as the mean \pm SD. The unpaired Student's t-test was used as appropriate to assess the statistical significance of the differences 


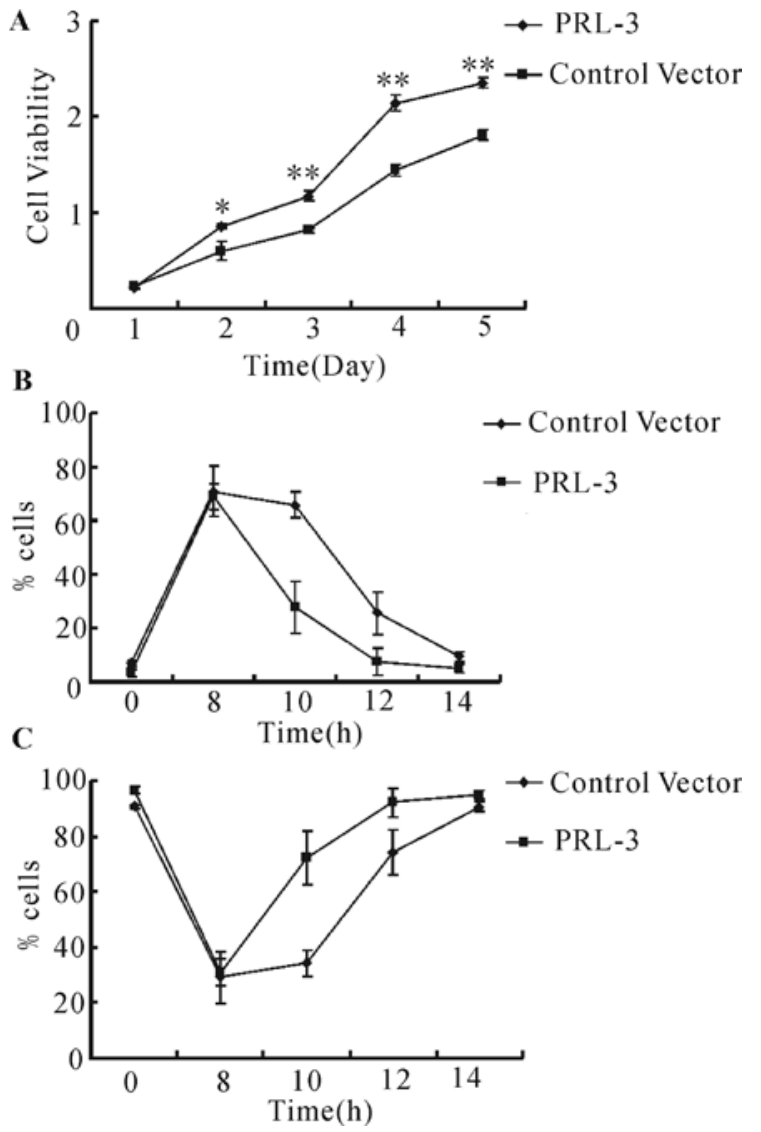

Figure 2. PRL-3 promotes the proliferation of LoVo cells and facilitates the G2/M transition. (A) Cells were trypsinized and seeded into a 96-well plate and cultured for 5 consecutive days. Cells growth was measured according to the protocols described in Materials and methods. The means and SD are representive of three independent experiments. ${ }^{*} \mathrm{p}<0.05,{ }^{* *} \mathrm{p}<0.01$, compared with control. Cells were synchronized at the G1/S boundary through double thymidine blockade. After the cells were released from the second thymidine blockade, the time was considered $0 \mathrm{~h}$ and the cell cycle distributions were analyzed by a FACS laser flow cytometer. The percentage of cells in the G2/M (B) and G1/S (C) phases of the cell cycle at the indicated timepoints were compared between control vector cells and PRL-3 cells.

between the two groups. Data about tumor growth in vivo or in vitro were analyzed by one way ANOVA. $\mathrm{p}<0.05$ was accepted as significant.

\section{Results}

Ectopic expression of PRL-3 promoted the growth of LoVo cells through $G 2$ and M phases. To investigate the mechanism through which PLR-3 promoted cell proliferation, LoVo cells were transfected with the PAcGFP-PRL-3 vector due to its low expression of endogenous PRL-3. After selection with G418, control vector cells and PRL-3 cells which were stably expressing ectopic PRL-3 were established(Fig. 1A). The expression of the ectopic PRL-3 was verified by both Western blotting (Fig. 1B) and real-time quantitative RT-PCR (Fig. 1C). Subsequently, we investigated the influence of PRL-3 expression on cell proliferation using the CCK-8 assay. Compared with the control vector cells, the proliferation of PRL-3 cells was promoted in a time-dependent manner (Fig. 2A). To further confirm the PRL-3-induced increase of cell proliferation and to explore which cell cycle phase was affected, flow
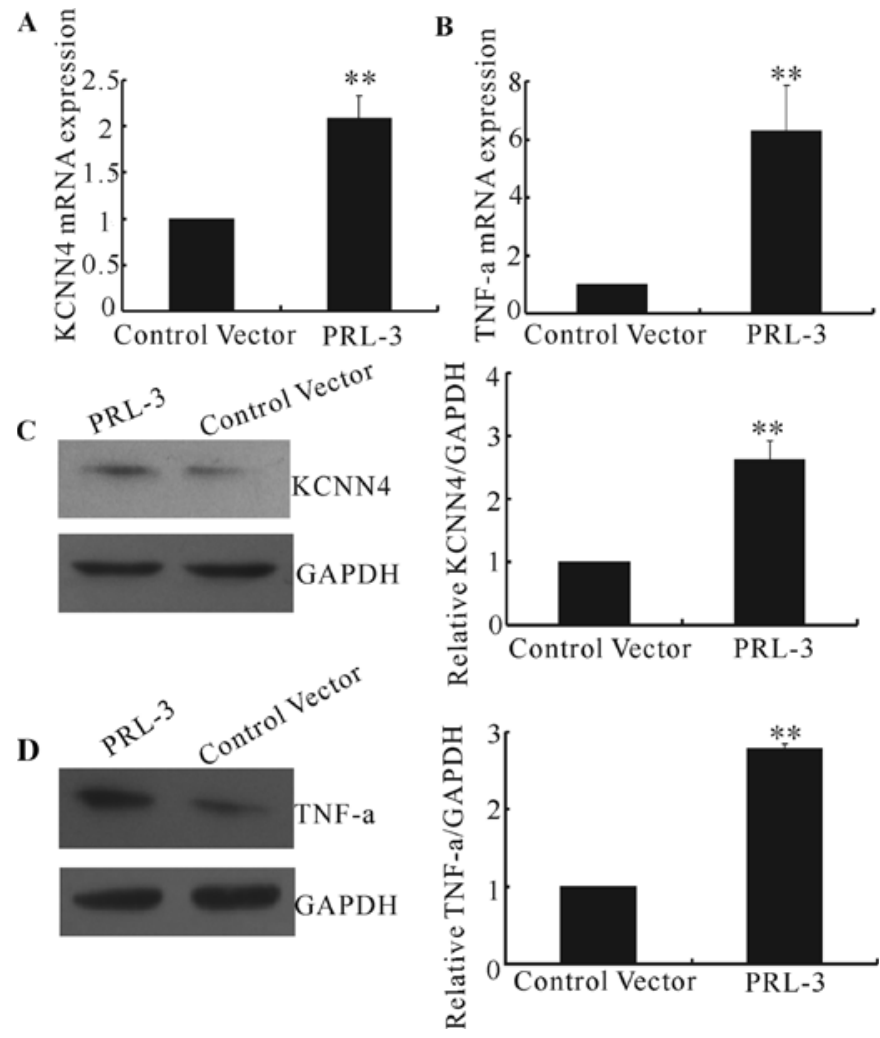

Figure 3. PRL-3 increases KCNN4 and TNF- $\alpha$ expression in LoVo cells. KCNN4 mRNA (A) and TNF- $\alpha$ mRNA (B) was determined by real-time quantitative RT-PCR, and GAPDH mRNA was used as an internal control. Representative and quantification immunoblot for KCNN4 (C) and TNF- $\alpha$ (D). GAPDH served as a loading control. The means and SD are representative of three independent experiments. ${ }^{* *} \mathrm{p}<0.01$, compared with control.

cytometry was used on synchronous LoVo cells transfected with control vector and PAcGFP-PRL-3 vector. Both control vector cells and PRL-3 cells were arrested by a double thymidine block at the G1/S phase transition and then released to allow cells to enter the $\mathrm{S}$ phase and to undergo mitosis. Results indicated that control vector cells and PRL-3 cells both exhibited their peak $\mathrm{G} 2 / \mathrm{M}$ phase at $8 \mathrm{~h}$ followed by exit from the G2/M phase and entry into the G1 phase. However, compared with PRL-3 cells, control vector cells showed a delayed exit from the G2/M phase and entry into the G1 phase by $2 \mathrm{~h}$ (Fig. 2B). According to the percentage of cells (\% cells) measured at the indicated timepoints, both of types of cells showed their peak $\mathrm{G} 2 / \mathrm{M}$ phase at 8 h, respectively $(71.1 \pm 9.4$ vs. $69.2 \pm 4.8)$. The percentage of control vector cells in the $\mathrm{G} 2 / \mathrm{M}$ phase was $65.8 \pm 4.9,25.6 \pm 7.9,9.3 \pm 1.6$ at 10,12 and $14 \mathrm{~h}$, respectively. In contrast, the percentage of PRL-3 cells in G2/M phase was $27.8 \pm 9.5,7.7 \pm 5.1,4.9 \pm 1.5$ at the corresponding timepoints, respectively. Conversely, the percentage of G1/S phase PRL-3 cells was more than that of the control vector cells starting at $8 \mathrm{~h}$ (Fig. 2C).

Upregulation of PRL-3 enhances the expression of KCNN4 dependent on the activation of $N F-\kappa B$. We have shown that PRL-3 promoted the growth of LoVo cells. To further explore the mechanism, we verified the expression of KCNN4 in PRL-3 cells and control vector cells, respectively. Real-time quantitative RT-PCR detected a much higher level of KCNN4 expression in LoVo cells transfected with PAcGFP-PRL-3 vector than in 

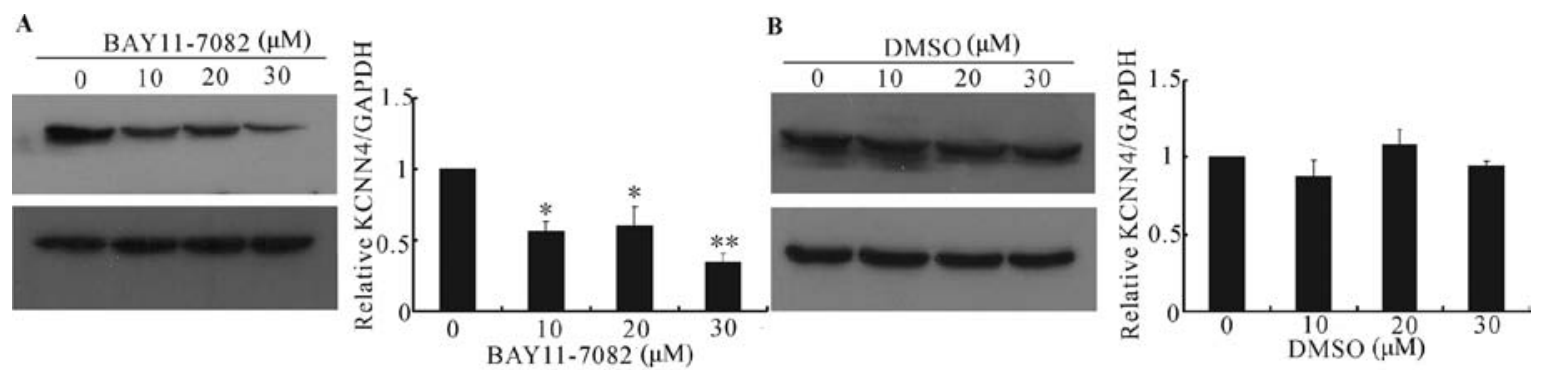

C
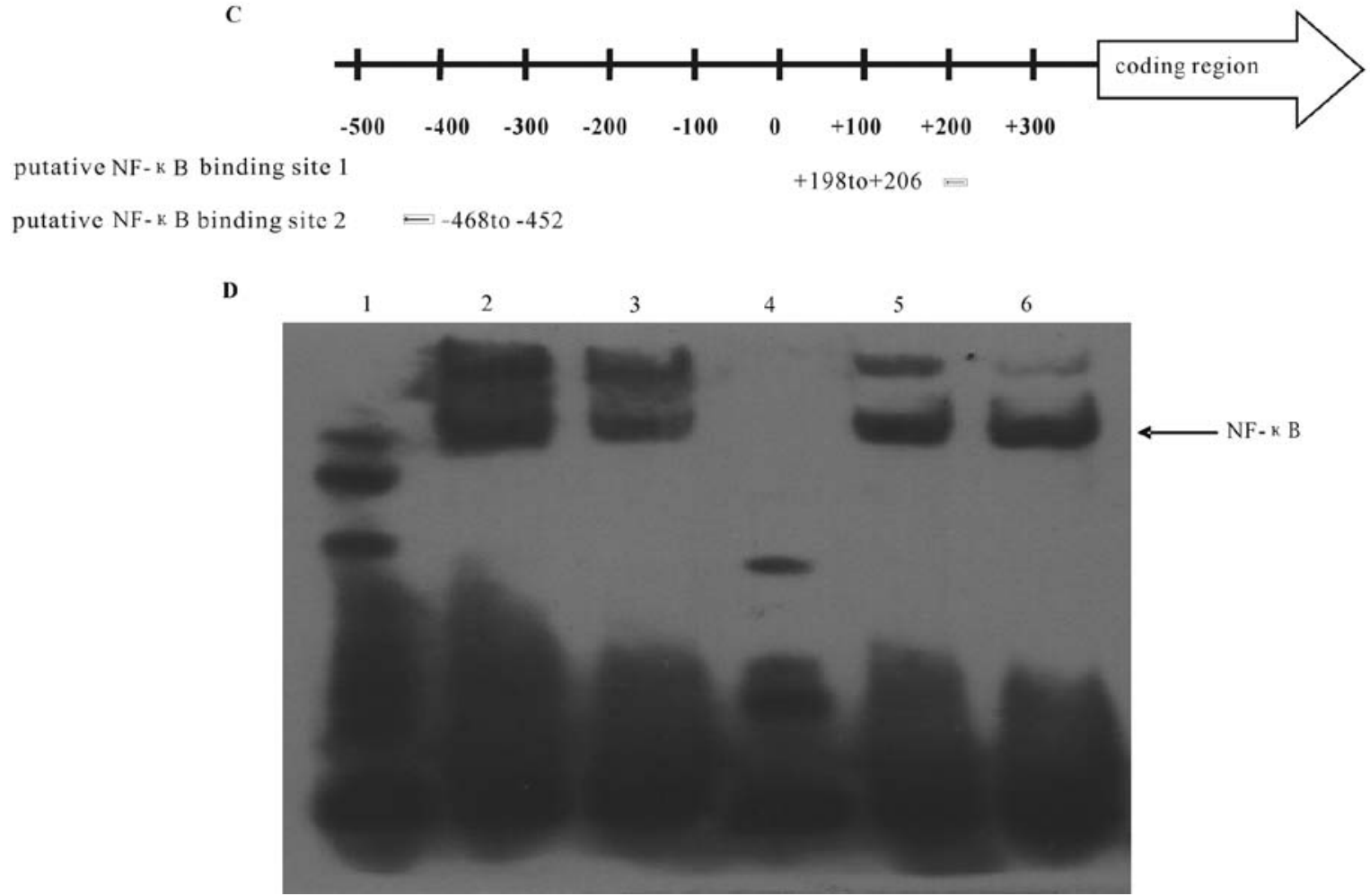

Figure 4. BAY11-7082 significantly inhibits the expression of KCNN4 induced by PRL-3. Cells were trypsinized and seeded for $24 \mathrm{~h}$, and then treated for 12 $\mathrm{h}$ with (A) BAY11-7082 and (B) DMSO at the indicated concentrations. Then the expression of KCNN4 was determined by Western blotting, GAPDH served as a loading control. The means and SD are representative of three independent experiments. ${ }^{*} \mathrm{p}<0.05,{ }^{* *} \mathrm{p}<0.01$, compared with control group. (C) Schematic description of the putative binding site 1 (antisense) and 2 (antisense) for NF-кB in the KCNN4 promoter region. (D) Biotin-labeled DNA probes were bound to nuclear proteins isolated from control vector cells (lanes 2 and 5) and PRL-3 cells (lanes 3 and 6). Lanes 1 and 4 served as the negative control for the two biotin-labeled putative NF- $\mathrm{\kappa B}$ binding probes 1 and 2, respectively.

cells transfected with control vector (Fig. 3A). Apart from this, we also verified the upregulation of KCNN4 at the protein level through Western blot analysis (Fig. 3C). We then investigated the mechanism by which the expression of KCNN4 channels was increased by PRL-3. Previous research revealed that the expression of small conductance $\mathrm{Ca}^{2+}$-activated $\mathrm{K}^{+}$channels $\left(\mathrm{K}_{\mathrm{Ca}} 2.2\right)$ were regulated by $\mathrm{TNF}-\alpha$ in an NF- $\kappa \mathrm{B}$-dependent manner (17). Here, we examined the effect of PRL-3 on the level of TNF- $\alpha$ in LoVo cells. Once cells were transfected with PAcGFP-PRL-3 vector, the expression of TNF- $\alpha$ was significantly elevated (Fig. 3B and D). To further explore whether the observed increase of the expression of KCNN4 channels was NF- $\mathrm{B}$ dependent, we used a specific inhibitor, BAY117082 , known to inhibit the activity of NF- $\mathrm{BB}$. As shown in Fig. 4A, compared with the control group, the expression of KCNN4 channels was significantly inhibited to $56.2 \%, 59.8 \%$ and $34.4 \%$ at the indicated concentrations of BAY11-7082, respectively. Considering the cytotoxicity of BAY11-7082, higher concentrations of BAY11-7082 were not used (data not shown). This observation suggested that inhibition of NF-кB prevented the expression of KCNN4 induced by PRL-3. To further demonstrate whether KCNN4 is a direct transcriptional target of $\mathrm{NF}-\kappa \mathrm{B}$, we scanned the KCNN4 genomic region and identified two putative NF-кB-binding sites (Fig. 4C). We then performed experiments using the electrophoretic mobility shift assay to determine whether NF- $\kappa \mathrm{B}$ can bind to the two putative $\mathrm{NF}-\kappa \mathrm{B}$-binding sites of the KCNN4 gene. As shown in Fig. 4D, biotin labeled two putative $\mathrm{NF}-\kappa \mathrm{B}$ binding probes 1 and 2 bound to unclear protein extracted from control vector cells (lanes 2 and 5) and PRL-3 cells (lanes 3 and 6), respectively. To explore whether the functional KCNN4 channels are present in LoVo cells, we used the KCNN4 channel activator 1-EBIO to elicit KCNN4 currents in the presence or not of TRAM-34 in the extracelluar medium. As shown in Fig. 5, 1-EBIO $(200 \mu \mathrm{M})$ significantly induced the $\mathrm{K}^{+}$current in the pipette solution with free calcium. Moreover, the 1-EBIO-induced currents were 


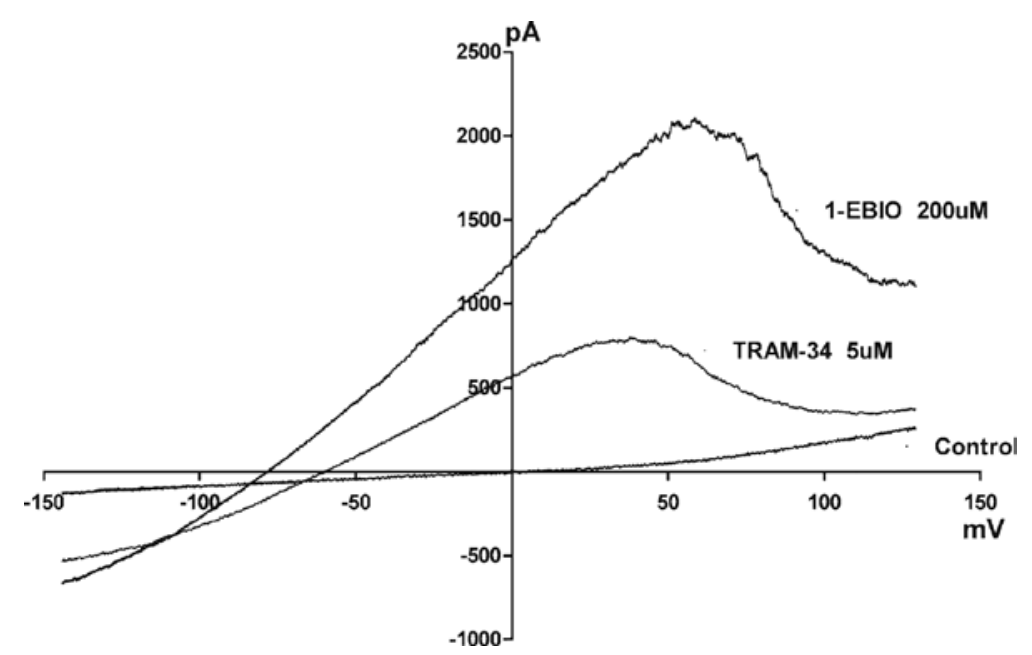

Figure 5. Functional expression of KCNN4 channels in LoVo cells. Ramp current traces were elicited by 250 msec voltage ramp from -150 to +150 mv. To confirm the functional KCNN4 in LoVo cells, KCNN4 currents were elicited by the application of the KCNN4 activator 1-EBIO (200 $\mu \mathrm{M})$ and then the specific inhibitor TRAM-34 was used to block the KCNN4 currents. Typical traces shown were obtained in a bath solution before and after application of $5 \mu \mathrm{M}$ TRAM-34.
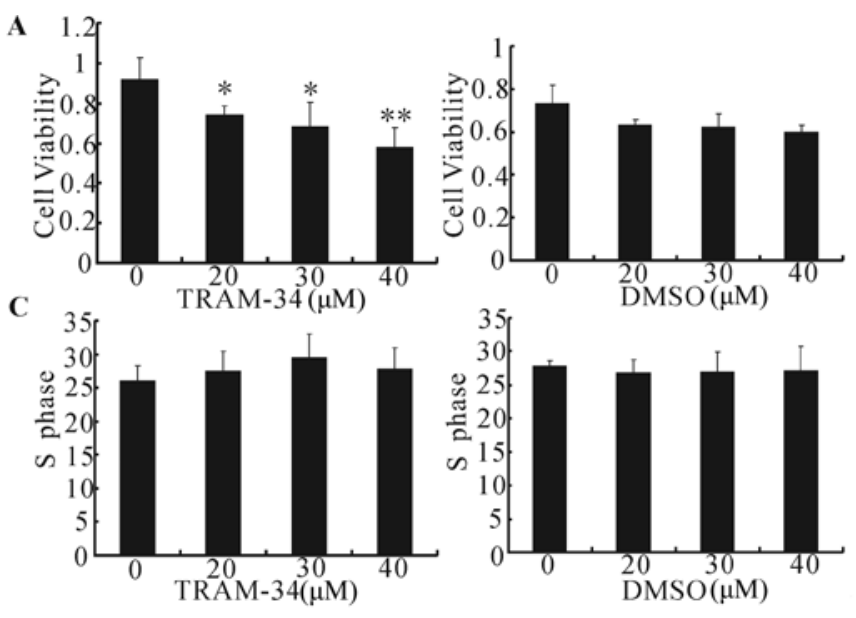

B
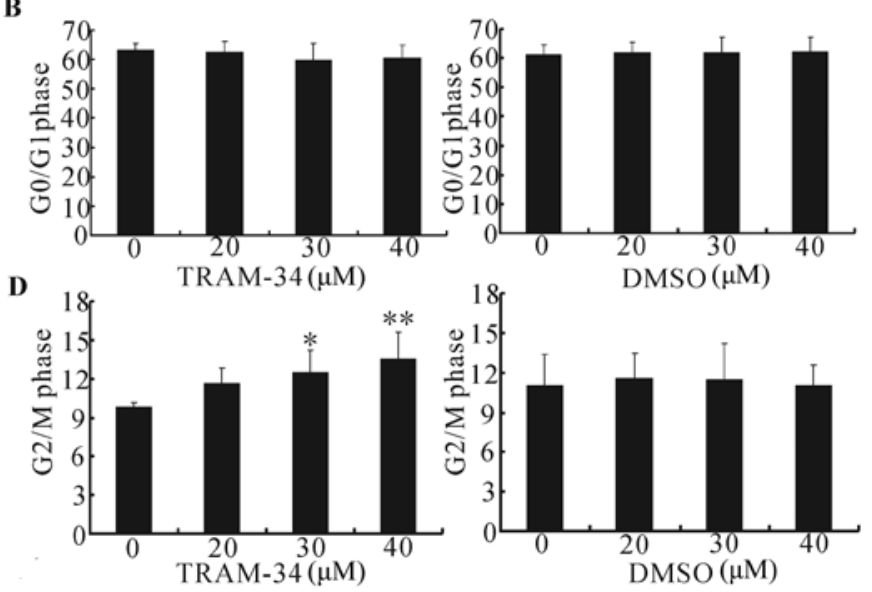

Figure 6. Blocking of KCNN4 channels significantly inhibits PRL-3 cell proliferation and arrested the cells in the G2/M phase. (A) Cells were trypsinized and seeded for $24 \mathrm{~h}$, and then treated for $24 \mathrm{~h}$ with TRAM-34 at the indicated concentrations. Cell growth was then measured according to protocols described in Materials and methods. The means and SD are representative of three independent experiments. ${ }^{*} \mathrm{p}<0.05,{ }^{* *} \mathrm{p}<0.01$, compared with the DMSO-treated control group. Quantitative analysis of PRL-3 cells (\% of the total) cell cycle distribution at the G0/G1 phase (B), S phase (C) and G2/M phase (D) treated by the indicated concentration of TRAM-34 for $24 \mathrm{~h}$ through a FACS laser flow cytometer. The means and SD are representative of three independent experiments. * $\mathrm{p}<0.05$, compared with the DMSO-treated control group.

greatly reduced by $5 \mu \mathrm{M}$ TRAM-34. These results demonstrate that functional KCNN4 channels were present in LoVo cells.

Blocking of KCNN4 prevents $P R L-3$-induced proliferation of LoVo cells through the G2/M phase transition. Considering the role of KCNN4 in the proliferation of cancer cells, we wondered whether the PRL-3-induced growth of LoVo cells was supported by upregulation of KCNN4. As shown in Fig. 6A, TRAM-34 in a dose-dependent manner inhibited the growth of PRL-3 cells. To further investigate how the KCNN4 channel inhibitor prevented cell proliferation, we measured the cell cycle progression using flow cytometry. There was a non-significant decrease in the number of cells in the G0/G1 phase (Fig. 6B) and a non-significant increase in the number of cells in S phase (Fig. 6C) in the presence of various concentrations of TRAM-34, which indicated that the inhibition of LoVo cell proliferation by blocking of the KCNN4 channels did not occur through the G1/S phase. At the same time, cells treated with various concentrations of TRAM-34 for $24 \mathrm{~h}$ resulted in a dose-dependent increase in the percentage of cells in G2/M phase of the cell cycle (Fig. 6D).

Blocking of KCNN4 channels induces the phosphorylation of $C d c 2$. To further confirm the effect of KCNN4 on the cell cycle of PRL-3 cells, phosphorylation of $\mathrm{Cdc} 2$, a protein involved in the regulation of the G2 phase and mitosis was examined. Since TRAM-34 was found to arrest PRL-3 cells in the G2/M phase transition compared with the DMSO-treated control group, we examined whether such phenomena involved the phosphorylation of the Thr14 and Tyr15 residues of Cdc2 considering their negative effect on the kinase activity of $\mathrm{Cdc} 2$. As shown in Fig. 7A, when cells were exposed to the indicated 


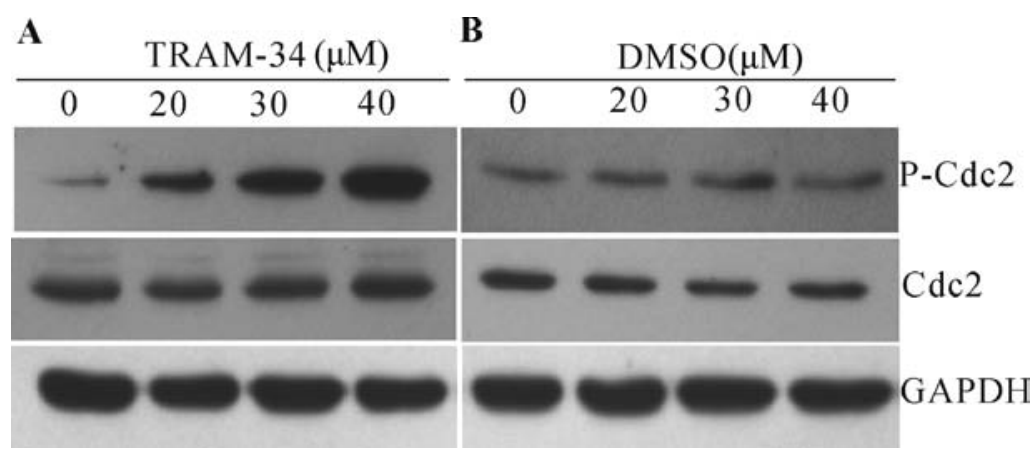

Figure 7. Blocking of KCNN4 channels induces the phosphorylation of Cdc2. (A) PRL-3 cells were treated with different concentrations of TRAM-34 for $2 \mathrm{~h}$. Western blot analyses were performed with anti-p-Cdc2 p34 (Thr14/Tyr15) and anti-Cdc2 p34 as described in Materials and methods. The phosphorylation of Cdc2 was also detected in DMSO-treated control group with the corresponding concentrations (B). GAPDH served as a loading control.
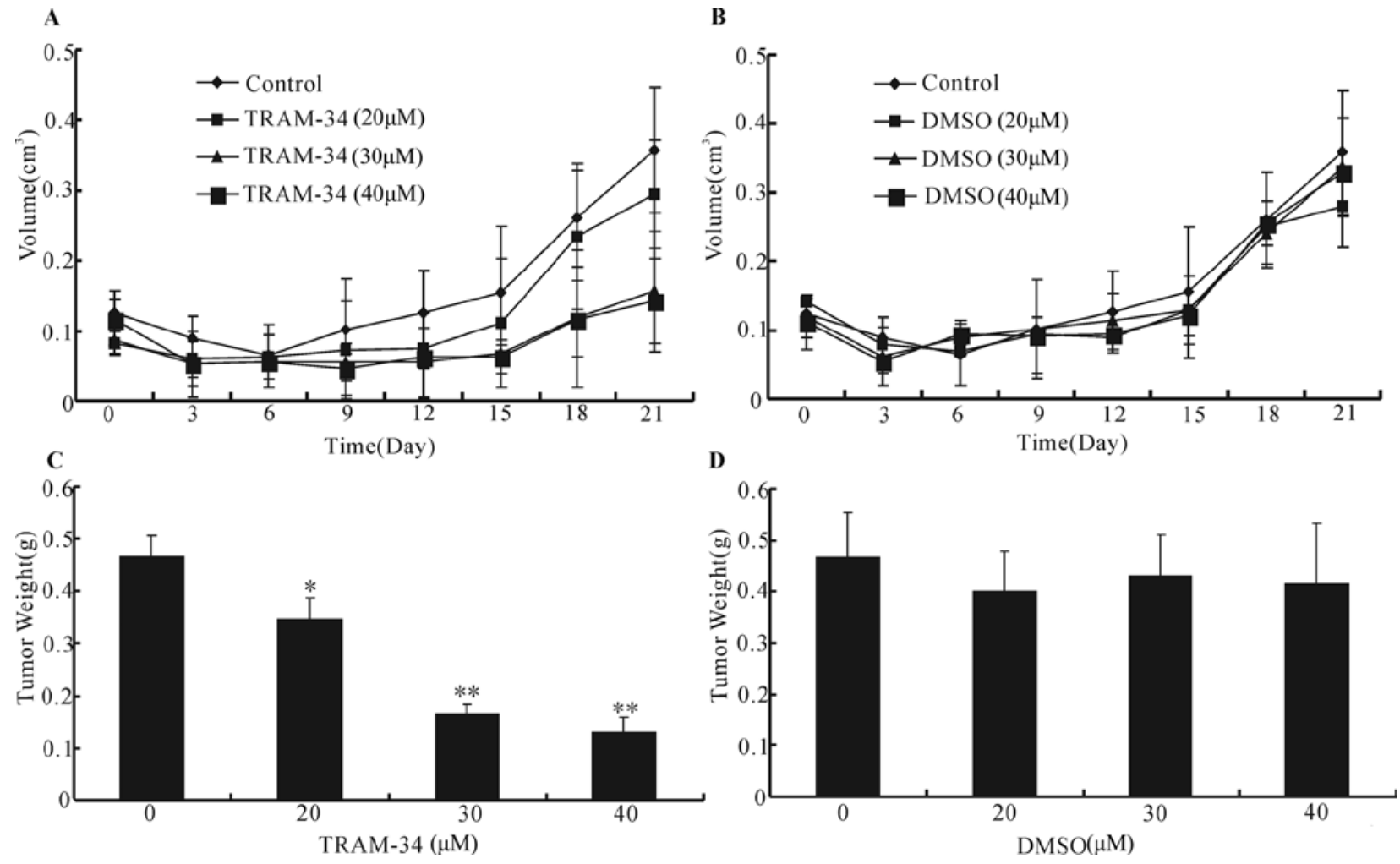

Figure 8. TRAM-34 at the indicated concentrations inhibits the growth of PRL-3 cells in nude mice. During the experiment and at the indicated days tumor volume (A and $\mathrm{B}$ ) and weight $(\mathrm{C}$ and $\mathrm{D})$ were measured. ${ }^{* *} \mathrm{p}<0.01,{ }^{*} \mathrm{p}<0.05$ compared with DMSO-treated control group.

concentrations of TRAM-34, the level of phosphorylation of Cdc2 was more remarkable compared with the DMSO-treated control group (Fig. 7B). The data suggest that KCNN4 induced the $\mathrm{G} 2 / \mathrm{M}$ phase cell cycle arrest via phosphorylation of $\mathrm{Cdc} 2$.

Blocking of KCNN4 channels prevents the tumor growth in nude mice. To further confirm that KCNN4 channels were critical for tumor development, PRL-3 cells treated with different concentrations of TRAM-34 and DMSO were injected into the right flank of nude mice. Tumor weight and volume were recorded at regular intervals. On Day 21, all nude mice were sacrificed and tumors were excised. The tumor weight and volume of the different groups treated with the indicated concentrations of TRAM-34 were compared. When treated with TRAM-34, tumor volumes were significantly smaller than the control group (Fig. 8A). At the end of the experiment, tumor weight was significantly lower in the TRAM-34-treated groups compared with the controls (Fig. 8C), However, in the DMSO-treated control group, there was no such difference in volume (Fig. 8B) and weight (Fig. 8D). These results indicate that KCNN4 plays an important role in the PRL-3 induced growth of LoVo cells in vitro.

\section{Discussion}

In present study, we have shown that the PRL-3 enhanced the proliferation of LoVo cells through the upregulation of KCNN4 channels. The specific inhibitor TRAM-34 was also used to 
further explore the role of KCNN4 channels in the proliferation of LoVo cells transfected with PRL-3. As a member of the protein-tyrosine phosphatases (PTPs) family, PRL-3 has a C-terminal prenylation (CCVM) motif and has been proven to have a close relationship with plasma membrane and endosomal structures (18). In our research, PRL-3 tagged with PAcGFP-C3 was observed to locate mainly at the cytoplasmic membrane of LoVo cells.

As mentioned above, KCNN4 channels are voltageindependent and sensitive to the change of intracellular $\mathrm{Ca}^{2+}$. However, the realization of such sensitivity depends on calmodulin which is bound to the KCNN4 channels in a $\mathrm{Ca}^{2+}$ independent manner (19). Openning of the KCNN4 channels leads to the efflux of $\mathrm{K}^{+}$ions, which could lead to hyperpolarization of the membrane potential, promotion of the $\mathrm{Ca}^{2+}$ influx and facilitation of the mitogenic activation. It is also well known that the concentration of $\mathrm{Ca}^{2+}$ in the cytoplasm is indispensable for the proliferation of cells (20). Given the unique role of KCNN4 channels, researchers have revealed that the KCNN4 channels participate in the growth of human endometrial cancer cells (21). Furthermore, overexpression of KCNN4 has been found in pancreatic cancer tissues (22). In our experiment, using the specific inhibitor TRAM-34 significantly inhibited the proliferation of LoVo cells transfected with the PAcGFP-PRL-3 vector in vivo and in vitro, respectively, which was consistent with a role of the KCNN4 channels mentioned above. This indicates that KCNN4 may participate in the cell proliferation induced by PRL-3.

Previous studies have attributed the upregulation of KCNN4 channels to the activation of extracellular signal-regulated kinases $($ ERK1/2) in vascular smooth muscle cells $(23,24)$ and that the NF- $\mathrm{KB}$ transcription factor critically regulates the expression of small conductance $\mathrm{Ca}^{2+}$ channels (25). In agreement with these findings, we have shown that expression of TNF- $\alpha$ was increased with transfection of the PAcGFP-PRL-3 vector. The NF-кB-specific inhibitor BAY11-7082 significantly inhibited the expression of KCNN4 in a dose-dependent manner. Furthermore, the electrophoretic mobility shift assay revealed that there were two NF- $\mathrm{KB}$ binding sites in the promoter region of KCNN4. Considering TNF- $\alpha$ as an acutocrine and paracrine growth factor (26), we demonstrated that upregulation of TNF- $\alpha$ induced by PRL- 3 was likely to be the mechanism of PRL-3-mediated increase of KCNN4 channels.

It has been known that $\mathrm{K}^{+}$channels strictly control the membrane potential which is essential for proliferation. The blockade of $\mathrm{K}^{+}$efflux inhibits the proliferation since transient hyperpolarization is essential for progression from the G1 into the $\mathrm{S}$ phase (27). On the contrary, activation of $\mathrm{K}^{+}$channels accelerates cell cycle progression from the G0/G1 into the $S$ phase (28). In the present study we examined whether the KCNN4-specific inhibitor TRAM-34 could result in cell progression arrest in G1/S phase. Surprisingly, flow cytometry results demonstrated that when cells were exposed at various concentrations of TRAM-34, the percentage of cell in the G1 and S phases was not significantly altered. However, the cell population in the $\mathrm{G} 2 / \mathrm{M}$ phase was dose-dependently increased. Similar results have been found in human airway smooth muscle (HASM) cells (29). Furthermore, we also used thymidine to synchronize cells at the G1/S phase in order to explore whether PRL-3 could influence the cell cycle at the
G2/M phase. Our data indicate that when PRL-3 was transfected into LoVo cells, although both control vector cells and PRL-3 cells showed peak G2/M phase at the same time, control vector cells had a delayed exit from the G2/M phase and entry into G1. Such results further indicated that PRL-3 promoted cell proliferation via regulation of the cell cycle $\mathrm{G} 2 / \mathrm{M}$ phase and not the G1/S phase.

As mentioned above, activation of KCNN4 could maintain the hyperpolarized membrane potential. Conversely, inhibition of KCNN4 could prevent $\mathrm{Ca}^{2+}$ entry into the cytoplasm which is required for cell growth and proliferation (30). Inhibition of the calcium/calmodulin pathway remarkably prevents the proliferation of MCF-7 human breast carcinoma cell line (31). Considering that TRAM-34 induced cell cycle arrest at G2/M phase, we examined the phosphorylation of the cell division control protein Cdc2 since dephosphorylation of Tyr15 and Thr14 is critical for cell entry into mitosis (32). As a target of Cdc25-C, the active Cdc25-C enzyme can activate the Cdc2cyclin B protein kinase in HeLa cells (33). However, further studies have suggested that the Cdc25-C activation is regulated by the $\mathrm{Ca}^{2+}$-dependent activation of the CaM kinase II (34). In our study, the anti-phospho-Cdc2 antibody revealed a significant increase in the level of $\mathrm{Cdc} 2$ phosphorylation when the KCNN4-specific inhibitor TRAM-34 was used, which supports the idea that upregulation of KCNN4 could increase the activation of $\mathrm{Cdc} 2$ facilitating the $\mathrm{G} 2 / \mathrm{M}$ phase transition.

In conclusion, we have shown that PRL-3 promoted proliferation through upregulation of KCNN4. Importantly, such proliferation was not dependent on the acceleration of the cell cycle progression from the G1 into the S phase but on the G2/M phase transition. The data obtained from using a specific inhibitor further supported our findings. A new role of KCNN4 was revealed in regulation of the cell cycle and a new mechanism by which PRL-3 promoted the proliferation of tumor cells was explored.

\section{Acknowledgements}

This study was supported by grants from the Science and Technology Project of Guangdong Province (no. 2007B031513012); and Natural Science Fund of Guangdong Province (no. 9151008901000058 and 10151008901000071).

\section{References}

1. Jemal A, Siegel R, Ward E, Hao Y, Xu J and Thun MJ: Cancer Statistics, 2009. CA Cancer J Clin 59: 225-249, 2009.

2. Jonas S, Thelen A, Benckert C, et al: Extended resections of liver metastases from colorectal cancer. Word J Surg 13: 511-521, 2007.

3. Saha S, Bardeli A, Buckhaults P, et al: A phosphatase associated with metastsasis of colorectal cancer. Science 294: 1343-1345, 2001.

4. Alonso A, Sasin J, Bottini N, et al: Protein tyrosine phosphatases in the human genome. Cell 117: 699-711, 2004.

5. Miskad UA, Semba S, Kato H, et al: High PRL-3 expression in human gastric cancer is a marker of metastasis and grades of malignancies: an in situ hybridization study. Virchows Arch 450: 303-310, 2007.

6. Fagerli UM, Holt RU, Holien T, et al: Overexpression and involvement in migration by the metastasis-associated phosphatase PRL-3 in human myeloma cells. Blood 111: 806-815, 2008.

7. Qian F, Li YP, Sheng X, et al: PRL-3 siRNA inhibits the metastasis of B16-BL6 mouse melanoma cells in vitro and in vivo. Mol Med 13: 151-159, 2007. 
8. Matsukawa Y, Semba S, Kato H, Koma Y, Yanagihara K and Yokozaki H: Constitutive suppression of PRL-3 inhibits invasion and proliferation of gastric cancer cell in vitro and in vivo. Pathobiology 77: 155-162, 2010.

9. Polato F, Codegoni A, Fruscio R, et al: PRL-3 phosphatase is implicated in ovarian cancer growth. Clin Cancer Res 11: 6835-6839, 2005.

10. Werner SR, Lee PA, DeCamp MW, Crowell DN, Randall SK and Crowell PL: Enhanced cell cycle progression and down regulation of $\mathrm{p} 21(\mathrm{Cip} 1 / \mathrm{Waf} 1)$ by PRL tyrosine phosphatases. Cancer Lett 202: 201-211, 2003.

11. Felipe A, Vicente R, Villalonga N, et al: Potassium channels: new targets in cancer therapy. Cancer Detect Prev 30: 375-385, 2006.

12. Spitzner M, Ousingsawat J, Scheidt K, Kunzelmann K and Schreiber R: Voltage-gated $\mathrm{K}^{+}$channels support proliferation of colonic carcinoma cells. FASEB J 21: 35-44, 2007.

13. Nehrke K, Quinn CC and Begenisich T: Molecular identification of $\mathrm{Ca}^{2+}$-activated $\mathrm{K}^{+}$channels in parotid acinar cells. Am J Physiol Cell Physiol 284: C535-C546, 2003.

14. Logsdon NJ, Kang J, Togo JA, Christian EP and Aiyar J: A novel gene, $\mathrm{hKCa} 4$, encodes the calcium-activated potassium channe in human T lymphocytes. J Biol Chem 272: 32723-32726, 1997.

15. Lallet-Daher H, Roudbaraki M, Bavencoffe A, et al: Intermediateconductance $\mathrm{Ca}^{2+}$-activated $\mathrm{K}^{+}$channels (IKCa1) regulate human prostate cancer cell proliferation through a close control of calcium entry. Oncogene 28: 1792-1806, 2009.

16. Qiao M, Shapiro P, Fosbrink M, Rus H, Kuma R and Passaniti A: Cell cycle-dependent phosphorylation of the RUNX2 transcription factor by cdc2 regulates endothelial cell proliferation. J Biol Chem 281: 7118-7128, 2006.

17. Dolga AM, Granic I, Blank T, et al: TNF-alpha-mediates neuro-protection against glutamate-induced excitotoxicity via NF-kappaB-dependent up-regulation of K2.2 channels. J Neurochem 107: 1158-1167, 2008.

18. Zeng Q, Si X, Horstmann H, Xu Y, Hong W and Pallen CJ: Prenylation-dependent association of protein-tyrosine phosphatases PRL-1, -2 , and -3 with the plasma membrane and the early endosome. J Biol Chem 275: 21444-21452, 2000.

19. Fanger CM, Ghanshan S, Logsdon NJ, et al: Calmodulin mediates calcium-dependent activation of the intermediate conductance KCa channel, IKCa1. J Biol Chem 274: 5746-5754, 1999.

20. Magnier-Gaubil C, Herbert JM, Quarck R, et al: Smooth muscle cell cycle and proliferation. Relationship between calcium influx and sarco-endoplasmic reticulum $\mathrm{Ca}^{2+} \mathrm{ATPase}$ regulation. J Biol Chem 271: 27788-27794, 1996.

21. Wang ZH, Shen B, Yao HL, Jia YC, Ren J, Feng YJ and Wang YZ: Blockage of intermediate-conductance- $\mathrm{Ca}^{2+}$-activated $\mathrm{K}^{+}$channels inhibits progression of human endometrial cancer. Oncogene 26 5107-5114, 2007

22. Jager H, Dreker T, Buck A, Giehl K, Gress T and Grissmer S: Blockage of intermediate-conductance $\mathrm{Ca}^{2+}$-activated $\mathrm{K}^{+}$channels inhibit human pancreatic cancer cell growth in vitro. Mol Pharmacol 65: 630-638, 2004
23. Si H, Grgic I, Heyken WT, Maier T, Hoyer J, Reusch HP and Köhler R: Mitogenic modulation of $\mathrm{Ca}^{2+}$-activated $\mathrm{K}^{+}$channels in proliferating A7r5 vascular smooth muscle cells. $\mathrm{Br} \mathrm{J}$ Pharmacol 148: 909-917, 2006.

24. Fioretti B, Castigli E, Micheli MR, et al: Expression and modulation of the intermediate-conductance $\mathrm{Ca}^{2+}$-activated $\mathrm{K}^{+}$ channel in glioblastoma GL-15 cells. Cell Physiol Biochem 18: 47-56, 2006.

25. Kye MJ, Spiess J and Blank T: Transcriptional regulation of intronic calcium-activated potassium channel SK2 promoters by nuclear factor-kappa B and glucocorticoids. Mol Cell Biochem 300: 9-17, 2007.

26. Wu S, Boyer CM, Whitaker RS, Berchuck A, Wiener JR, Weinberg JB Jr and Bast RC: Tumor necrosis factor alpha as an autocrine and paracrine growth factor for ovarian cancer: monokine induction of tumor cell proliferation and tumor necrosis factor alpha expression. Cancer Res 53: 1939-1944, 1993.

27. Ghanshani S, Wulff $\mathrm{H}$, Miller MJ, et al: Up-regulation of the IKCa1 potassium channel during T-cell activation. Molecular mechanism and functional consequences. J Biol Chem 275: 37137-37149, 2000.

28. Chittajallu R, Chen Y, Wang H, et al: Regulation of Kvl subunit expression in oligodendrocyte progenitor cells and their rolein G1/S phase progression of the cell cycle. Proc Natl Acad Sci USA 99: 2350-2355, 2002.

29. Shepherd MC, Duffy SM, Harris T, et al: $\mathrm{KCa} 3.1 \mathrm{Ca}^{2+}$ activated $\mathrm{K}^{+}$channels regulate human airway smooth muscle proliferation. Am J Respir Cell Mol Biol 37: 525-531, 2007.

30. Thebault S, Flourakis M, Vanoverberghe K, et al: Differential role of transient receptor potential channels in $\mathrm{Ca}^{2+}$ entry and proliferation of prostate cancer epithelial cells. Cancer Res 66: 2038-2047, 2006.

31. Rodriguez-Mora OG, LaHair MM, McCubrey JA and Franklin RA: Calcium/calmodulin-dependent kinase I and calcium/calmodulin-dependent kinase kinase participate in the control of cell cycle progression in MCF-7 human breast cancer cells. Cancer Res 65: 5408-5416, 2005.

32. Marraccino RL, Firpo EJ and Roberts JM: Activation of the p34 CDC2 protein kinase at the start of $S$ phase in the human cell cycle. Mol Biol Cell 3: 389-401, 1992.

33. Hoffmann I, Clarke PR, Marcote MJ, Karsenti E and Draetta G: Phosphorylation and activation of human cde25-C by cdc2cyclin B and its involvement in the self-amplification of MPF at mitosis. EMBO 12: 53-63, 1993.

34. Patel R, Holt M, Philipova R, Moss S, Schulman H, Hidaka H and Whitaker M: Calcium/calmodulin-dependent phosphorylation and activation of human $\mathrm{Cdc} 25-\mathrm{C}$ at the $\mathrm{G} 2 / \mathrm{M}$ phase transition in HeLa cells. J Biol Chem 274: 7958-7968, 1999. 